\title{
Exercise Counseling
}

National Cancer Institute

\section{Source}

National Cancer Institute. Exercise Counseling. NCI Thesaurus. Code C101235.

A process by which a health professional creates a regimen of physical activity intended to maintain or improve the health of a subject. (ACC) 